\title{
Tecnología en discapacidad: Una aproximación en la terminología ${ }^{1}$
}

\author{
Technology in disability: an approach in terminology
}

\author{
Diana Carolina Rincón Martínez ${ }^{2}$ \\ Johana Paola Ríos Ortegón ${ }^{3}$ \\ Mónica Romero Espinosa ${ }^{4}$
}

Recibido: Febrero 202014 • Enviado para modificación: Mayo 132014 • Aceptado: Junio 202014.

Rincón, D.; Ríos J.; Romero M. (2014). Tecnología en discapacidad: Una aproximación en la terminología. Revista Ocupación Humana, 14 (1), pp. 29-40.

Resumen: Esta revisión bibliográfica busca reconocer y establecer cuáles son los principales términos utilizados en el área de tecnología en discapacidad y su posible utilidad para el caso colombiano. En primer lugar, se analiza el concepto de tecnología y su evolución histórica en el campo de la rehabilitación-discapacidad; por otro lado, se realiza un recorrido por los conceptos comúnmente usados, los cuales fueron encontrados en diferentes estudios relacionados con la utilización de aparatos o dispositivos de baja complejidad en personas con discapacidad (1995-2013), obteniendo información a partir de las principales revistas científicas de Terapia Ocupacional y Rehabilitación de países como Estados Unidos, Canadá, España, Reino Unido, Colombia, Brasil, entre otros, con el fin de realizar un análisis de esta terminología y las corrientes predominantes (Americana y Europea). Por último, se discute si en Colombia existe un término específico que facilite la comprensión y clasificación de los diferentes aparatos o apoyos tecnológicos en el campo de la discapacidad.

Palabras clave: Tecnología, persona con discapacidad, dispositivos de autoayuda.

Abstract: This article is a bibliographic review that searches to recognize and establish
which are the main terms used in the area of technology in disability and its possible utility
for the Colombian case. In first place, it is analyzed the concept of technology and its
historical evolution in the field of the rehabilitation-disability, in the other hand it is done a
tour around the concepts commonly used, which were found in many studios related with
the using of apparatus or devices of low complexity in persons with disability (1995-2003)
getting information from the main journals of Occupational Therapy and Rehabilitation

\footnotetext{
${ }^{1}$ Artículo resultado de la investigación que las autoras desarrollan en su proyecto de grado, Guía para la prescripción, diseño y elaboración de ayudas técnicas de baja complejidad en la actividad de alimentación, para optar por el título de terapeutas ocupacionales en la Universidad Nacional de Colombia, bajo la dirección de la profesora asociada al Departamento de la Ocupación Humana, Sylvia Cristina Duarte Torres, de la Universidad Nacional de Colombia.

${ }^{2}$ Estudiante de Terapia Ocupacional. Universidad Nacional de Colombia. dicrinconma@unal.edu.co

${ }^{3}$ Estudiante de Terapia Ocupacional. Universidad Nacional de Colombia. jopriosor@unal.edu.co

${ }^{4}$ Estudiante de Terapia Ocupacional. Universidad Nacional de Colombia. mromeroe@unal.edu.co
} 
of countries as U.S.A, Canada, Spain, United Kingdom, Colombia, Brazil, among others, in order to do an analysis of this terminology and the prevailing currents (American and European). Finally, it is discussed if in Colombia exists a specific term that facilitates the comprehension and classification of the many apparatus or technological supports in the disability field.

Key words: Technology, disabled persons, assistive technology, assistive device, self-help devices.

\section{Introducción}

Actualmente la tecnología es una parte esencial de la vida de los seres humanos, al ser utilizada como una herramienta para simplificar el trabajo de las personas en diferentes contextos. Así mismo, existe un área de la tecnología que está pensada a favor de las personas con discapacidad, en la cual, a través de modificaciones, innovaciones o creaciones, se promueve la participación de los usuarios que presentan limitaciones en la ejecución de diferentes actividades. Este desarrollo de la tecnología ha sido conocido desde la prehistoria, hasta el siglo XXI.

Diversas definiciones se han venido configurando a través de los años para comprender el significado del término tecnología, cada una de las cuales aporta nociones que acercan el contenido a una realidad. Según la Real Academia Española (1984), tecnología se define como "el conjunto de conocimientos propios de un oficio mecánico o arte industrial, además de ser el lenguaje propio exclusivo técnico de una ciencia o arte" (p. 1291).

Para el año 2008, la tecnología se concibe como un "conjunto de conocimientos propios de una técnica y un conjunto de instrumentos, procedimientos o recursos técnicos emplea- dos en un determinado sector o producto" (García, 2008, p. 965); mientras que en el año 2012, se reconoce como una "disciplina científica que estudia la técnica en la aplicación práctica de una ciencia o de una actividad especializada" (Real Academia Nacional de Medicina, 2012, p. 1570).

Para estas definiciones se establece como común denominador, el conocimiento técnico aplicado en diferentes áreas. Tal como afirman Cook \& Hussey (2002), ninguna de estas definiciones menciona algo sobre los dispositivos y para tal efecto, se reconocen diferentes términos que se han venido utilizando indistintamente para identificar aquellos productos creados, modificados o innovados para las personas con discapacidad.

En los referentes históricos se reconocen todos aquellos dispositivos creados para facilitar el desempeño ocupacional de personas que presentan o pueden llegar a presentar alguna restricción en la participación. Desde el área de rehabilitación, se habla específicamente de dispositivos diseñados en principio para corregir, suplir funciones, facilitar tareas o mejorar el desempeño.

Durante la prehistoria, se registran algunos hallazgos, los cuales sitúan la 
utilización de diferentes dispositivos en personas que lo requirieran. Según Salinas, Lugo \& Restrepo (2008), en momias del antiguo Egipto se encontraron órtesis y otros dispositivos para el tratamiento de algunas discapacidades. En el año 4000 a.C. se hallaron aditamentos similares a muletas, $y$ cerca del año 2380 a.C., se reconocen imágenes en las cuales aparece representada una persona que utiliza un bastón con apoyo en la axila (Zambudio, 2009). Para el siglo XVI, el desarrollo de la tecnología en discapacidad se relaciona fuertemente con la rehabilitación física, así como con el diseño y fabricación de órtesis y prótesis.

El desarrollo de la tecnología en discapacidad según Viosca (1999), Humphrey \& Holzapfer (2002) (citados por Zambudio, 2009), está relacionado con los grandes acontecimientos bélicos. Por ejemplo, después de la I y II Guerra Mundial se incrementa la investigación y surgen los primeros laboratorios e instituciones destacados en el diseño y la construcción de los diferentes dispositivos ortoprotésicos.

En las últimas décadas, el diseño y la elaboración de productos de ayuda para las personas que presentan algún tipo de limitación en su participación, se ha acogido a los recientes conocimientos de diferentes tecnologías, apropiando la electrónica, la robótica y la domótica. Todas estas, desarrolladas para el mejoramiento del desempeño de las personas, tal como se observa en dispositivos como sillas de ruedas, en las cuales se despliega una gran variedad de alternativas, desde simples componentes mecánicos, hasta complejos circuitos electrónicos que favorecen la independencia en la movilidad.

Es importante rescatar que "algunas de las disciplinas que han ido conformando grupos multidisciplinarios son: la medicina, la ingeniería, la ortopedia, la biomédica, el diseño, y la arquitectura, entre muchas otras que trabajan conjuntamente en el diseño de ayudas técnicas para ofrecer un alto grado de autonomía" (Sevilla, 2011, p. 29).

El desarrollo tecnológico ha permitido mejorar la calidad de vida de las personas, a partir de la satisfacción de las necesidades que se generan día a día. La tecnología en discapacidad y rehabilitación surge como un área pensada a favor de las personas que presentan alguna restricción en la participación, con el fin de facilitar o mejorar las habilidades requeridas para la ejecución de una actividad cotidiana, favoreciendo así el desempeño en la comunidad.

No obstante, al revisar los principales autores e investigadores relacionados con tecnología en discapacidad, se observa que utilizan distintos términos para referirse a este tipo de tecnología, sin mostrar diferencias claras entre unos y otros o evidenciar la complejidad de la tecnología utilizada.

Por ello, este documento tiene como objetivo presentar una revisión de la literatura de las concepciones planteadas en tecnología en discapacidad durante el periodo comprendido entre 1995 y 2013. Esta revisión, tiene en cuenta diversos autores quienes han publicado sus evidencias en revistas de Terapia Ocupacional y Rehabilitación, con el propósito de proveer conocimiento en el área de tecnología y 
Terapia Ocupacional, así como esclarecer la procedencia y definición de los términos utilizados.

Se analizan los diferentes términos para hacer un acercamiento a uno solo, que abarque la función y finalidad de los diferentes dispositivos tecnológicos de baja complejidad usados por las personas con discapacidad. Por último se discute, para el caso colombiano, cómo la aproximación a un término específico facilita la comprensión y clasificación de diferentes aparatos tecnológicos en el campo de la discapacidad.

\section{Método}

Se realiza una revisión de textos publicados entre los años 1995 y 2013; se incluyen artículos científicos, trabajos de grado y textos nacionales e internacionales que cumplen con los criterios de búsqueda: textos propios de Terapia Ocupacional, literatura en español, inglés y portugués, ya que en estos idiomas se encuentra la mayor producción científica en Terapia Ocupacional; a partir del uso de términos clave: assistive technology, assistive device, technical aid, assitive aids adaptive equipment.

En una primera fase se realizó un acercamiento a los trabajos de grado de cinco universidades que ofrecen el programa de Terapia Ocupacional en Bogotá y Cali: Universidad Nacional de Colombia - Sede Bogotá, Universidad del Rosario, Universidad Manuela Beltrán, Escuela Colombiana de Rehabilitación y Universidad del Valle.

La segunda fase de la búsqueda abarcó revistas de Terapia Ocupacional:
Revista Ocupación Humana, Revista Chilena de Terapia Ocupacional, Revista Terapia Ocupacional Galicia, Revista de Terapia Ocupacional da Universidade de São Paulo, The American Journal of Occupational Therapy, Canadian Journal of Occupational Therapy, British Journal of Occupational Therapy, Australian Occupational Therapy Journal, Scandinavian Journal of Occupational Therapy y The Indian Journal of Occupational Therapy.

En la última fase se llevó a cabo la búsqueda en siete bases de datos: Biblioteca Virtual en Salud, Academic Search Complete, Embase, Medline, Normas Técnicas Colombianas, Scielo y Science Direct; y revistas de rehabilitación: Revista Colombiana de Medicina Física y Rehabilitación; Assistive Technology: The Official Journal; Journal of Rehabilitation, Research \& Development y Technology and Disability.

\section{Resultados}

Esta revisión arrojo diez términos relacionados con tecnología en discapacidad, específicamente con dispositivos de baja complejidad empleados comúnmente por profesionales del área de la salud, principalmente en Terapia Ocupacional (Tabla 1).

Para el reconocimiento de la terminología se establecen dos corrientes, la americana y la europea, considerando para la primera los países: Canadá, Estados Unidos, Colombia, Chile, Argentina y Brasil, de los cuales se revisaron 20 artículos científicos.

En la segunda corriente se incluye el Reino Unido, España y los Países Es- 
Tabla 1. Términos comúnmente usados en tecnología de discapacidad

\begin{tabular}{|l|l|l|}
\hline \multicolumn{1}{|c|}{ Términos } & \multicolumn{1}{|c|}{ Traducción al español } & $\begin{array}{c}\text { Número de documentos } \\
\text { consultados }^{\mathbf{5}}\end{array}$ \\
\hline Assistive technology & $\begin{array}{l}\text { Tecnología de asistencia/tecnología } \\
\text { asistida }\end{array}$ & 17 \\
\hline Assistive device & $\begin{array}{l}\text { Dispositivo de asistencia /dispositivo } \\
\text { asisitivo }\end{array}$ & 13 \\
\hline Technical aid & Ayuda técnica & 4 \\
\hline Adaptive equipment & Equipamiento adaptado & 2 \\
\hline Adaptations & Adaptación & 1 \\
\hline Adaptive technology & Tecnología adaptada & 1 \\
\hline Assistive aids & Ayudas de asistencia & 1 \\
\hline Dispositivos de tecnología en asistencia & $\begin{array}{l}\text { Dispositivos de tecnología en } \\
\text { asistencia }\end{array}$ & 1 \\
\hline Equipo asistencial & Equipo asistencial & 1 \\
\hline Sistemas adaptados & Sistemas adaptados & 1 \\
\hline
\end{tabular}

Fuente. Elaboración propia.

candinavos: Noruega, Suecia y Dinamarca; también se consideran India y Australia, pues si bien estos países no pertenecen al continente europeo, presentan similitudes en la utilización de terminología asociada a tecnología en discapacidad. Estos países se escogieron debido al mayor número de publicaciones científicas relacionadas con la temática a tratar; de la región europea se revisaron un total de 23 artículos.

La Figura 1 da cuenta de los términos comúnmente usados en la literatura consultada y los periodos de tiempo en los cuales han sido utilizados. La figura comprende una línea del tiempo desde 1995 a 2013 y dos recuadros, uno de la corriente americana y otro de la europea. En cada uno de ellos pueden apreciarse los principales términos usados: tecnología de asistencia, dispositivos de asistencia y ayuda técnica, los cuales son recurrentes en las dos corrientes, salvo el término sistemas adaptados referido en 1995 por la Disability Discrimination Act, y un término reciente, dispositivo de tecnología de asistencia, referido en Colombia por Losada (2007).

Cabe aclarar que en la figura no se hace distinción de los países que usan cada término y tampoco a qué hacen referencia específicamente. Sin embargo, se presentará una revisión de los conceptos teniendo en cuenta estos parámetros.

\section{Corriente americana}

Dentro de esta categoría se evidencia la utilización de diversos términos para dar cuenta del manejo de diferentes dispositivos en personas con algún tipo de discapacidad; se establece en la corriente americana el uso constante del término tecnología de asistencia o en inglés assistive technology (Buning,

\footnotetext{
${ }^{5}$ Corresponde al número de artículos publicados entre 1995 y 2013 en los cuales aparece el término referido.
} 
Figura 1. Utilización de términos de tecnología en discapacidad desde 1995 a 2013

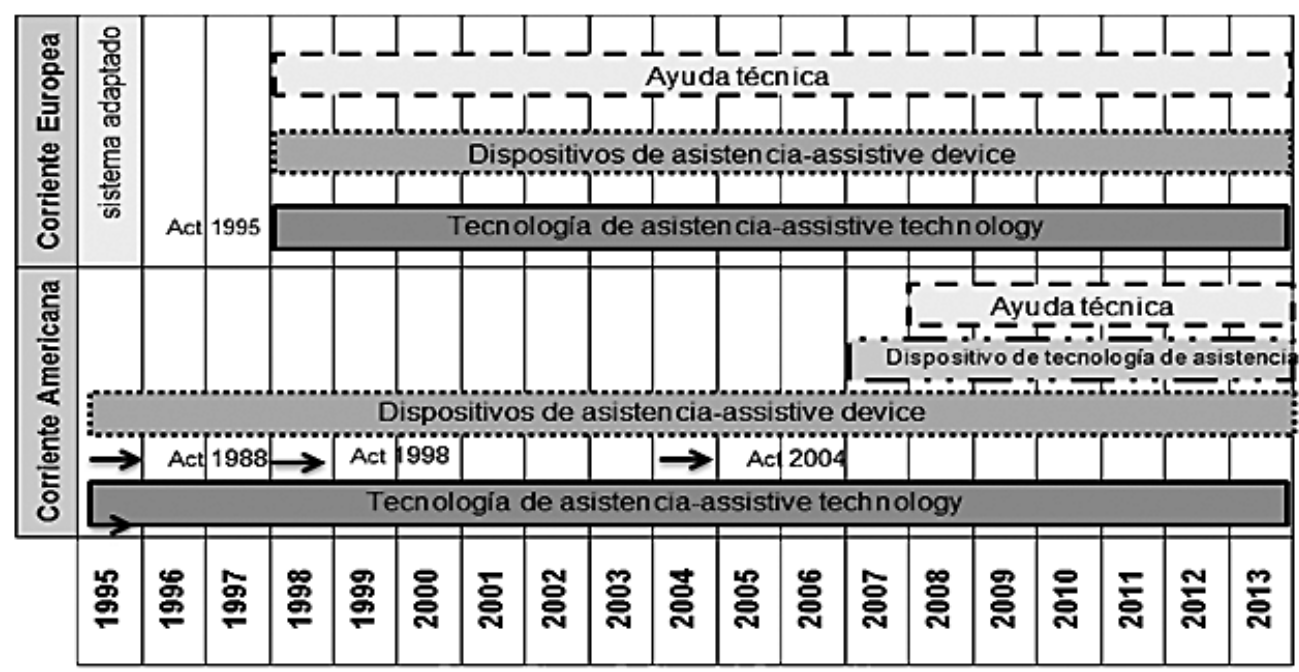

Fuente. Elaboración propia.

Hammel, Schmeler, \& Doster, 2004), que antes del año 1995, fue definida en Technology-Related Assistance for Individuals with Disabilities Act of 1988 como "cualquier objeto, pieza de equipo, sistema de productos, ya sea adquirido comercialmente, modificado o personalizado, que se utiliza para aumentar, mantener o mejorar las capacidades funcionales de las personas con discapacidad" (p. 98).

El concepto planteado es recurrente en el ACT-1988, reformada en 1998 y 2004 (Technology - Related Assistance for Individuals with Disabilities Act Amendments, 1998; The Assistive Technology Act, 2004), donde no existe variación alguna en la definición, manteniéndose la generada en 1988 en Estados Unidos.

Es importante explicar que el concepto assitive technology lo acogen en países como Canadá, Brasil y Chile, evi- denciado en autores como Finlayson, Guglielmello \& Liefer (2001); Cook \& Hussey (2002); Buning, Hammel, Schmeler \& Doster (2004); Miller (2006) y Polgar (2006); y que en Brasil, en la Legislación Federal Básica de 2000, se retoma el concepto tecnología de asistencia consignado en el ACT-1998, así lo presentan autores como Rocha \& Castiglioni (2005); Alburquerque, Cezar Da Cruz, Jesús \& Agostini (2010); Cezar Da cruz, Toyoda \& Agostini (2012) y Guillaumon, Caires, Cezar Da Cruz \& Agostini (2013).

Entre tanto, se destaca la definición que se enuncia en la Clasificación Internacional del Funcionamiento, de la Discapacidad y de la Salud - CIF como "cualquier producto, instrumento, equipamiento o tecnología, adaptada especialmente y proyectada para mejorar la funcionalidad de una persona" (OMS, 2001, p.187), retomada de la Clasificación de ayudas técnicas ISO 
9999 (segunda edición) que para el caso Colombiano sigue la última actualización (1994), en la cual se usa el término ayudas técnicas, aunque en otros países se considere el de productos de apoyo.

Estos conceptos se nutren de otras afirmaciones que apoyan la función de la tecnología de asistencia como:

- Una forma de compensación por medio de equipamiento especial y procedimientos modificados a las personas que no tienen la capacidad biomecánica para desempeñar de la manera usual las tareas de la vida cotidiana, de ocio y del trabajo. Se utiliza para salvar la distancia entre las capacidades del sujeto y las exigencias de la tarea (Kielhofner, 2005).

- Incorporar características de accesibilidad como "eludir las barreras ambientales" (Hedrick, Bender, Heinemann, Ruddell, \& Reis, 2006, p. 186), maximizar la independencia, mejorar el funcionamiento con el propósito de aumentar, mantener o mejorar las habilidades funcionales y la participación de la actividad de las personas con discapacidades (CAOT Position Statement, 2012).

Por otro lado, se encuentra el concepto dispositivo de asistencia -assistive device-, el cual es referido como sinónimo de tecnología de asistencia y definido de la misma forma, tal como lo plantean los autores Mann, Hurren \& Tomita (1995); Finlayson, Guglielmello \& Liefer (2001) y Steggles (2004). Cabe señalar la alusión de Petterson, Appelros \& Ahltrom (2007), quienes refieren que los dispositivos de asistencia son herramientas para vivir que pueden mejorar la calidad de vida y permitir la independencia en el diario vivir de personas con discapacidades.

\section{Corriente europea}

En esta región se identifica una gran variedad de términos usados en tecnología en discapacidad: sistemas adaptados, dispositivos de asistencia, ayudas tecnológicas, equipo adaptado, tecnología de asistencia, dispositivo de asistencia, equipo asistencial, adaptaciones, ayudas técnicas, entre otros. En su recorrido histórico, se destaca en Europa un primer momento situado en 1995, cuando se consolida la norma Disability Discrimination Act, en la cual se retoma el concepto sistemas adaptados, haciendo alusión al impulso para la implementación de los mismos. Otros autores como Hawkins \& Stewart (2002), refieren el término adaptación, como formas destinadas a mejorar la accesibilidad, específicamente en el ambiente del hogar.

Otro término utilizado es equipamiento adaptable -adaptive equipment-, referido en estudios de autores como Brittle, Lett, Littlechild \& Sackley (2007) y Wielandt \& Strong (2000) como cualquier dispositivo o sistema que permita a un individuo desempeñarse en una tarea; algunos dispositivos incluidos son sillas de ruedas, ayudas para el área de autocuidado, entre otras. Así mismo, autores como Schaik (2000) se refieren a la tecnología adaptable -adaptive technology-, como un término amplio que engloba tanto la tecnología de asistencia como la tecnología adaptada. 
Por otro lado, en la región escandinava autores como Klint, Harms-Ringdahl \& Seiger (1998) y Shipham \& Pitout (2003), retoman conceptos propios de la literatura americana como dispositivos de asistencia, refiriéndose a dispositivos disponibles para las personas con limitaciones funcionales o discapacidades, en lugar de cualquier equipo técnico diseñado para la población general (Löfqvist, Nygren, Széman \& Iwarsson, 2005).

De igual manera, en artículos publicados en el British Journal of Occupational Therapy se retoma el concepto de dispositivos de asistencia, definiéndolos como "piezas portables de equipamiento que pueden ser instaladas o removidas por personal de Terapia Ocupacional con o sin conocimientos técnicos" (Wilson, McCracken \& Cummings, 1999, p. 269) y "cualquier equipo de ayuda que permita la participación de las personas con discapacidad en una actividad deseada" (Hawkins \& Stewart, 2002, p.81). Entre tanto, Hopkins y Smith (1993, p. 326 citado por Shipham \& Pitout, 2003), retoman el concepto del ACT1988, que define la tecnología de asistencia. A partir de este concepto, se destaca el permitir la independencia de personas con discapacidad o adultos mayores (Percival, 2012).

El siguiente término encontrado en la literatura científica consultada corresponde a tecnología de asistencia -assistive technology-, referido por diferentes autores desde 1998 hasta la actualidad como Chamberlain, Evans, Neighbour \& Hughes (2001); Goodacre \& Turner (2005); Goodacre, McCreadie, Flanagan \& Lans- ley (2008), basados en la Comisión Real de Atención a Largo Plazo (Royal Commission on Long Term Care), la cual define tecnología de asistencia como un término general para "cualquier dispositivo o sistema que permite a un individuo realizar una tarea que de otro modo no podría hacer, aumentado la facilidad y la seguridad con la cual puede ser desempeñada" y como "la forma más común de atención a largo plazo a partir de la utilización de algún tipo de equipamiento que no requiera cooperación o coordinación de otros".

Algunas investigaciones retoman varios conceptos para referirse a los dispositivos, tal es el caso de autores como Vincent (2000), quien usa los términos dispositivos de asistencia y tecnología de asistencia como sinónimos, y Löfqvist, Nygren, Széman, \& Iwarsson (2005), quienes especifican que la legislación utiliza el término ayudas técnicas. Sin embargo, se prefiere la utilización de dispositivo de asistencia, porque es un término comúnmente usado en Terapia Ocupacional, tal como se observa en la Tabla 1.

Entre tanto, cabe reconocer que entre las definiciones planteadas por los diferentes autores, varían en gran manera los tipos de aparatos tecnológicos a los cuales se hace referencia, empleando el término dispositivo de asistencia tanto para adaptaciones al ambiente (Logan et. al., 2009) como en la descripción de algunos tipos de órtesis (Shashidhar \& Manora, 2007).

\section{Discusión}

Los términos usados con mayor frecuencia por los profesionales de Tera- 
pia Ocupacional y de Rehabilitación son en su orden: tecnología de asistencia/ tecnología asistida -assistive technology-; dispositivo de asistencial dispositivo asistivo -assistive device-, y ayuda técnica -technical aid-; los demás términos identificados obtuvieron frecuencias iguales o menores a dos.

Los dos primeros términos han estado presentes en las dos corrientes, americana y europea, desde los inicios del área de la tecnología pensada para las personas con discapacidad. Para la corriente americana, a partir de la creación y divulgación del Technology - Related Assistance for Individuals with Disabilities Act of 1988 y para la corriente europea, hacia el año 1998. Cabe resaltar que en las dos corrientes, ambos términos han tenido un comportamiento paralelo y una vigencia durante el periodo de estudio (1995 - 2013), lo que apoya lo postulado por autores como Mann, Hurren \& Tomita (1995) y Finlayson, Guglielmello \& Liefer (2001), quienes consideran que los términos assistive technology y assistive device, son usados como sinónimos.

Cabe resaltar que aunque el desarrollo tecnológico en discapacidad se dio primero en la corriente americana (1988), el término technical aid, es relativamente nuevo en esta literatura, siendo empleado en textos a partir del año 2009; mientras tanto en la corriente europea, este sitúa su origen en el año 1998. Esto se puede relacionar con lo encontrado en la literatura americana, donde el mayor desarrollo tecnológico en el área de la discapacidad se sitúa en la década del 90, mientras se observa una disminución en el número de investigaciones y publicaciones en el área de rehabilitación física para el siglo XXI. Entre tanto en la región europea, se evidencia una creciente investigación en este campo, apoyada por los diversos términos desarrollados y utilizados por los profesionales de la salud; de igual modo, se observa que los términos europeos están permeando la corriente americana, lo que justifica su aparición reciente en la literatura de esta región.

A partir de la aproximación a la literatura propia de Terapia Ocupacional y Rehabilitación que tiene en cuenta la utilización de dispositivos de baja complejidad, se determina que si bien la terminología empleada para referirse a tecnología en discapacidad difiere según el país, el autor y diferentes aspectos sociopolíticos, en general los vocablos empleados buscan referirse a los dispositivos que facilitan o mejoran las habilidades necesarias para desempeñar una actividad cotidiana. La evidencia muestra que en la corriente americana se han realizado esfuerzos por regular el uso de términos en cuanto a tecnología en discapacidad, esto se refleja en la legislación, donde se consigna la terminología y conceptos que se ratifican en los diferentes documentos de Estado. Aun así, tanto en la corriente europea como en la americana se siguen utilizando indistintamente las expresiones relacionadas con tecnología de baja complejidad, dando prioridad a términos como assistive device, assistive technology o technical aid.

En el caso colombiano, al ser un país suramericano, la literatura referente a tecnología en discapacidad es ob- 
tenida principalmente de la corriente americana; ya que la producción bibliográfica contempla aspectos socio demográficos que aplican al contexto del país, en los autores colombianos que se afilian al tema de tecnología es posible observar la tendencia a seguir la terminología propuesta por esta COrriente. Esto lo reafirman autores como Sánchez \& Laserna (2002); Rincón, Ortiz \& Patiño (2005); Cerquera, Parada \& Robles (2005); Lizarazo (2012), al referirse a los diferentes aparatos tecnológicos dirigidos a las personas con discapacidad como dispositivos de tecnología en asistencia, así mismo autores como Ríos, Laserna, Melo \& Vargas (2007), quienes apoyan sus investigaciones en la Norma Técnica Colombiana NTC-ISO 9999-1994 que adopta el término ayudas técnicas, definiéndolas como "cualquier producto, instrumento, equipo o sistema técnico utilizado por una persona con limitación, fabricado especialmente o disponible en el mercado, para prevenir, compensar, mitigar o neutralizar la deficiencia, incapacidad o impedimento", siendo este el concepto utilizado con mayor frecuencia en los textos científicos colombianos en el campo de la discapacidad.

\section{Conclusiones}

La tecnología en discapacidad ha permitido facilitar el desempeño ocupacional de personas que presentan alguna restricción en la participación; se habla específicamente de dispositivos diseñados en principio para corregir, suplir funciones, facilitar tareas o mejorar el desempeño (área de rehabilitación). La misma, ha ido evolucionando de acuerdo a las necesidades de las personas, apropiándose a su vez del desarrollo y el avance científico, lo que ha permitido hacer más sofisticados diversos aparatos, para así satisfacer las metas de independencia, autonomía y calidad de vida.

Por esta razón, en el contexto de la rehabilitación y la tecnología, se vio la necesidad de darle un nombre propio a todos aquellos dispositivos diseñados o adaptados a favor de las personas con discapacidad. De acuerdo a esto, se han dispuesto legislaciones en las cuales se reivindica la importancia de utilizar dispositivos que favorezcan el desempeño de aquellas personas que presentan una limitación para interactuar con el ambiente. Sin embargo, la literatura científica de los últimos 18 años muestra que si bien la terminología empleada para referirse a tecnología en discapacidad difiere según el país, el autor y diferentes aspectos sociopolíticos, siempre llega a un punto en común, que es facilitar el desempeño ocupacional.

Lo anteriormente expuesto, deja en evidencia la necesidad de generar reglamentaciones o disposiciones que contemplen una terminología uniforme donde se describan claramente los términos asociados a la tecnología, para facilitar así la comprensión por parte de los profesionales y la comunidad en general respecto al tema, además de permitir la interpretación similar de conceptos por parte de todos los actores relacionados con la tecnología en discapacidad y de esta manera, orientar hacia las mismas metas la investigación, las políticas, la participación social y demás ámbitos en los que ésta esté inmersa. 


\section{Referencias}

Alburquerque, A., Cezar Da Cruz, D., Jesús, A. \& Agostini, R. (2010). Tecnología asistida en Brasil: reflexiones. TOG (A Coruña) revista internet. 7 (12). Disponible en: http://www.revistatog.com/num12/pdfs/ original5.pdf.

Brittle, N., Lett, K., Littlechild, R. \& Sackley, C. (2007). The Disability Profile of Adults who Receive Adaptive Equipment from Local Authority Occupational Therapy Services. British Journal of Occupational Therapy. 70 (11), 479-482.

Buning, M., Hammel, J., Schmeler, M. \& Doster, S. (2004). Assistive Technology within Occupational Therapy Practice (2004). The American Journal of Occupational Therapy. 58 (6), 678-680.

CAOT. Position statement. Assistive technology and Occupational Therapy. (2012) Canadian Journal of occupational therapy.

Cerquera, C.; Parada, A.; \& Robles, M. (2005). Base de datos en dispositivos de Tecnología de Asistencia para alimentación disponibles en Bogotá. Tesis de pregrado. Universidad Manuela Beltrán. Colombia. Disponible en: http://unicornio.umb.edu. co/tesis_terocup/2005/1105\%20C416.pdf.

Chamberlain, E., Evans, N., Neighbour, K \& Hughes, J. (2001). Equipment: is it the answer? An audit of equipment provision. British Journal of Occupational Therapy. 64(12), 595-600.

Cook, A. \& Hussey, S. (2002). Assistive technologies. Principles and practice. Estados Unidos: Mosbt, Inc.

Da Cruz, D., Toyoda, C. \& Agostini, R. (2012) Revisión de literatura sobre adaptación de bajo costo para el desempeño de actividades de la vida diaria. TOG (A Coruña) revista en internet. 9 (15). Disponible en: http://www.revistatog.com/num15/pdfs/ original6.pdf.

Finlayson, M., Guglielmello, L. \& Liefer, K. (2001). Describing and Predicting the Pos- session of Assistive Devices among Persons with Multiple Sclerosis. American Journal of Occupational Therapy. 55 (5), 545-551.

García, T. (2008). El pequeño Larousse ilustrado. México: Ediciones Larousse S.A.

Goodacre, K., McCreadie, C., Flanagan S. \& Lansley, P. (2008) Enabling Older People to Stay at Home: the Costs of Substituting and Supplementing Care with Assistive Technology. British Journal of Occupational Therapy. 71 (4), 130-140.

Goodacre, L. \& Turner, G. (2005). An Investigation of the Effectiveness of the Quebec User Evaluation of Satisfaction with Assistive Technology via a Postal Survey. British Journal of Occupational Therapy. 68 (2), 93-96.

Guillaumon, M., Caires, D., Cezar Da cruz, D \& Agostini, R. (2013) Catálogos de productos de tecnología asistiva para adaptaciones de la vida diaria: utilización de los terapeutas ocupacionales. TOG (A Coruña) revista en internet. 10 (17). Disponible en: http:/www.revistatog.com/num17/ pdfs/original18.pdf

Hawkins, R. \& Stewart, S. (2002). Changing Rooms: The impact of adaptations on the meaning of home for a disabled person and the role of Occupational Therapists in the process. British Journal of Occupational Therapy. 65 (2), 81-87.

Hedrick, B., Bender, T., Heinemann, A., Ruddell, J. \& Reis, J. (2006). Employment issues and assistive technology use for persons with spinal cord injury. Journal of Rehabilitation Research \& Development [Versión electrónica]. 43 (2). Disponible en: http://www.rehab.research.va.gov/ jour/06/43/2/pdf/Hedrick.pdf

Hopkins \& Smith (1993). En Shipham, M. \& Pitout, B (2003) Rheumatoid arthritis: hand function, activities of daily living, grip strength and essential assistive devices. Curationis, 98-105.

Humphrey \& Holzapfer (2002) En Zambudio, R. (2009). Prótesis, órtesis y ayudas técnicas. Barcelona: Elsevier Masson. 
Kielhofner, G. (2005). Conceptual Foundations of Occupational Therapy (3. $\cdot \underline{\mathrm{a}} \mathrm{Ed})$. Pp.6-74. Buenos Aires: Medica Panamericana.

Klint, C., Harms-Ringdahl, K. \& Seiger, A. (1998). Lift/transfer and technical aids for persons with severe acquired brain injury. Scand J Caring Sci. 12, 154-159.

Lizarazo, D. (2012). Dispositivos de tecnología de asistencia para el cuidado y protección personal que existen a nivel distrital para facilitar el desempeño ocupacional de personas que presentan parálisis generadas por el plexo braquial. (Tesis de grado). Terapia ocupacional. Universidad Manuela Beltrán. Colombia.

Löfqvist, C., Nygren, C., Széman, Z. \& Iwarsson, S. (2005). Assistive devices among very old people in five European countries. Scandinavian Journal of Occupational Therapy. 12,181 -192.

Logan, P., Murphy, A., Drummond, A., Bailey, S., Radford, K., Gladman, J. ,Conroy, S. (2007). An Investigation of the Number and Cost of Assistive Devices used by Older People who had fallen and Called a 999 Ambulance. British Journal of Occupational Therapy. 70 (11), 475-478.

Losada, A. (2007). Dispositivos de tecnología de asistencia para alimentación. TOG (A Coruña) [revista en Internet] (5). Disponible en: http://www.revistatog.com/ num5/pdfs/ORIGINAL3.pdf
Mann, W., Hurren, D. \& Tomita, M. (1995). Assistive Devices Used by Home-Based Elderly Persons with Arthritis. The American Journal of Occupational Therapy. 49 (8), 810-820.

Miller, J. (2006). Assistive technology as an enabler to occupation: what's old is new again. Canadian Journal of Occupational Therapy. 73 (4), 199-204.

Norma Técnica Colombiana- ISO 99991994 (1994). Ayudas técnicas para personas con limitación- Clasificación. Bogotá, Colombia: ICONTEC.

Organización Mundial de la Salud. (2001). Clasificación Internacional del Funcionamiento, de la Discapacidad y de la Salud. Disponible en: http://www.conadis.salud. gob.mx/descargas/pdf/Clasificacion-CIF. pdf

Parliament of the United Kingdom. (1995). Disability Discrimination Act 1995. Disponible en: http://www.legislation.gov.uk/ ukpga/1995/50/contents

Percival, J. (2012). Introducing daily living products to older people with sight loss: practice implication of an innovate demonstration programme. British Journal of Occupational Therapy. [Versión electrónica]. 75(12), 570-573. Disponible en: http://www.ingentaconnect.com/search/ar ticle?option $1=$ tka \&value $1=$ british + journ al+occupational+therapy \&pageSize $=10$ \&index $=1$ 